\title{
Incidencia de la COVID-19 en la pobreza, Región Huánuco, 2020
}

\author{
Incidence of COVID-19 on poverty, Huánuco Region, 2020
}

Milward Ubillus « 1,a, Janet Trujillo-Alvarez ${ }^{1, b}$, Jose Ubillús-Trujillo ${ }^{2, c}$, Irma Palacios Zevallos ${ }^{1, d}$, José Antonio Beraún Barrantes ${ }^{1, e}$

\author{
Filiación y grado académico \\ 1 Universidad César Vallejo, Trujillo, Perú. \\ 2 Instituto Nacional de Enfermedades \\ Neoplásicas, Lima, Perú. \\ a Magíster en Epidemiología Clínica. \\ b Obstetra. \\ c Economista. \\ d Doctora en Ciencias de la Salud. \\ e Médico cirujano.
}

(iD) ORCID iD de Milward Ubillús

https://orcid.org/0000-0002-3684-9394

(iD) ORCID iD de Janet Trujillo-Alvarez https://orcid.org/0000-0001-9494-1001

(iD) ORCID iD de Jose Ubillús-Trujillo https://orcid.org/0000-0002-1523-2571

(ID) ORCID iD de Irma Palacios Zevallos https://orcid.org/0000-0003-4163-8740

(iD) ORCID iD de José Beraún Barrantes https://orcid.org/0000-0001-8979-2734

Contribución de autoría

MU: diseño del estudio, redacción y aprobación del artículo científico.

JTA: diseño del estudio y ejecución del trabajo de campo.

JUT: diseño del estudio y ejecución del trabajo de campo.

IPZ: elaboración del informe final y redacción del artículo científico.

JABB: elaboración del informe final y redacción del borrador del artículo científico.

Fuentes de financiamiento

La investigación fue autofinanciada.

Conflictos de interés

Los autores declaran no tener conflicto de interés en la publicación de este artículo.

Recibido: 31-03-2021

Arbitrado por pares

Aceptado: 22-06-2021

Citar como

Ubillus M, Trujillo-Alvarez J, Ubillús-Trujillo J, Palacios Zevallos I, Beraún Barrantes JA. Incidencia de la COVID-19 en la pobreza, Región Huánuco, 2020. Rev Peru Cienc Salud. 2021; 3(3): $180-5$. doi: https://doi.org/10.37711/ rpcs.2021.3.3.350

Correspondencia

Milward Ubillús

Email: milubi@hotmail.com

\section{RESUMEN}

Objetivo. Determinar la relación entre la incidencia de la COVID-19 y la pobreza en la Región Huánuco durante el 2020. Métodos. Fue un estudio descriptivo, ecológico de grupo múltiple, transversal y retrospectivo. La población estaba compuesta por las 11 provincias y 84 distritos geopolíticos del departamento de Huánuco. El tipo de muestreo fue no aleatorio, intencionado por conveniencia. Se usaron datos de fuentes primarias, como el Reporte de Casos Nuevos de COVID-19 de la Dirección Regional de Salud - Huánuco - DIRESA, 2020. Resultados. El número de casos nuevos de COVID-19, las provincias de Huánuco, Leoncio Prado y Puerto Inca fueron las que presentaron el mayor número de casos, mientras que las provincias que presentaron el menor número de nuevos casos fueron Lauricocha, Yarowilca y Huacaybamba. Asimismo, las provincias de Puerto Inca, Leoncio Prado y Huánuco fueron las que presentaron las más altas tasas de incidencia. Al evaluar la pobreza en el departamento de Huánuco se tomó en cuenta la clasificación de los niveles de pobreza de cada distrito realizada al 2018 en el Mapa de Pobreza Monetaria Provincial y Distrital 2018 del INEI. La provincia de Leoncio Prado tiene los distritos más pobres, seguido de la provincia de Huánuco y Lauricocha. Las provincias que tienen los distritos menos pobres son Huacaybamba, Pachitea y Marañón. Conclusiones. Existe correlación positiva estadísticamente significativa de 0,646 para una $p<0,05$ entre el nivel de pobreza y la incidencia de la Covid-19 en Huánuco, durante el 2020.

Palabras clave: COVID-19; pobreza; Huánuco; coronavirus (fuente: DeCSBIREME).

\section{ABSTRACT}

Objective. To determine the relationship between the incidence of COVID-19 and poverty in the Huánuco Region during 2020. Methods. It was a descriptive, ecological, multiple group, cross-sectional and retrospective study. The population consisted of the 11 provinces and 84 geopolitical districts of the department of Huánuco. The type of sampling was non-random, purposive by convenience. Data from primary sources were used, such as the Report of New Cases of COVID-19 of the Regional Health Directorate - Huanuco DIRESA, 2020. Results. Regarding the number of new cases of COVID-19, the provinces of Huánuco, Leoncio Prado and Puerto Inca had the highest number of cases, while the provinces with the lowest number of new cases were Lauricocha, Yarowilca and Huacaybamba. Likewise, the provinces of Puerto Inca, Leoncio Prado and Huánuco had the highest incidence rates. When evaluating poverty in the department of Huanuco, the classification of poverty levels in each district carried out as of 2018 in INEl's 2018 Provincial and District Monetary Poverty Map was taken into account. The province of Leoncio Prado has the poorest districts, followed by the province of Huánuco and Lauricocha. The provinces with the least poor districts are Huacaybamba, Pachitea and Marañón. Conclusions. There is a statistically significant positive correlation of 0.646 for a $p<0.05$ between the level of poverty and the incidence of COVID-19 in Huánuco, during 2020.

Keywords: COVID-19; poverty; Huánuco; coronavirus (source: MeSH NLM). 


\section{INTRODUCCIÓN}

La última gran y actual pandemia vivida por la humanidad se inició en China, el 31 de diciembre de 2019, en que fueron reportados 27 casos de neumonía de origen desconocido en una ciudad Ilamada Wuhan ${ }^{(1,2)}$. La enfermedad presentaba un cuadro clínico con fiebre, tos $y$ disnea, siendo su complicación más grave la neumonía (3). Una semana después del reporte, el Centro Chino para el Control y la Prevención de Enfermedades descubrió que la causa de la enfermedad era un nuevo coronavirus (SARS-CoV-2) que más tarde la OMS denominó COVID-19 del acrónimo inglés Coronavirus Disease $2019^{(1,4)}$. Los datos epidemiológicos reportaban que en el $80 \%$ de la población, esta epidemia pasaría inadvertida o con síntomas leves, en el $15 \%$ se presentaría con complicaciones que serían resueltas por el sistema de salud, pero en un $5 \%$ aproximadamente sí sería muy grave y complicada, pudiendo terminar en la muerte ${ }^{(5,6)}$. La expansión de la enfermedad se dio de una manera muy rápida a nivel global, llegando a nuestro país y confirmándose el primer caso el 6 de marzo de 2020. El segundo y tercer caso de COVID-19 en el Perú se presentaron en Huánuco, en dos hermanos de 21 y 15 años provenientes de Europa, siendo confirmados e informados a la opinión pública por el Ministerio de Salud el 10 de marzo de $2020^{(7)}$. A partir de entonces se inició la propagación de la enfermedad a nivel regional, nacional y mundial, registrándose casos nuevos de manera diaria, siendo el departamento de Huánuco uno de los más afectados dadas las difíciles condiciones sanitarias, educativas y sociales de su población ${ }^{(8)}$.

Como consecuencia de la amenaza de esta nueva pandemia, el Gobierno peruano dictó una serie de medidas para controlar la diseminación del virus, siendo una de ellas la cuarentena total en el país, con la consecuente inamovilidad. Está fue una medida muy discutida pues pondría en peligro la economía del país, al detener el movimiento económico de todos los sectores productivos.

En cuanto a la pobreza, el 17 de octubre de 2020, el secretario general de las Naciones Unidas explicó que las personas más pobres son las que corren mayor riesgo de contagiarse del virus y las que tienen menos acceso a una atención de salud de calidad. "Para las personas más pobres del mundo, la pandemia de covid-19 representa una doble crisis", advirtió ${ }^{(9)}$.

Considerando los registros de la Dirección Regional de Salud - DIRESA Huánuco sobre incidencia de COVID-19 2020 y el Mapa de Pobreza Monetaria Provincial y Distrital 2018 elaborado por Instituto Nacional de Estadística e informática - INEI, la presente investigación se planteó como objetivo determinar la relación entre la incidencia de COVID-19 y la Pobreza en Huánuco durante el 2020.

\section{MÉTODOS}

\section{Tipo de estudio}

Se realizó un estudio descriptivo, ecológico de grupo múltiple, transversal y retrospectivo.

\section{Población y muestra}

La población considerada para el estudio fueron las 11 provincias y sus 84 distritos geopolíticos del departamento de Huánuco, siendo la unidad de análisis cada distrito del departamento, agrupados por cada provincia ${ }^{(13)}$. Se usó el tipo de muestreo no aleatorio, intencionado por conveniencia.

\section{Instrumentos de recolección de datos}

Se utilizó un instrumento de recolección de datos validado por juicio de expertos de la investigación. Se usaron datos de fuentes primarias, como el Reporte de Casos Nuevos de COVID-19 de la Dirección Regional de

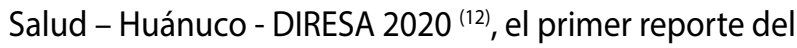
2021 y el último Mapa de Pobreza Monetaria Provincial y Distrital 2018 del Instituto Nacional de Estadística e Informática - INEI.

\section{Análisis de datos}

Se usaron las medidas de asociación como la tasa de incidencia. Para la contrastación de la hipótesis se usó la prueba de correlación de Spearman dado que se evaluó una variable numérica y una variable ordinal. Los datos fueron registrados en el instrumento de recolección de datos, luego fueron digitados en una base de datos Excel y posteriormente se evaluaron con el Software STATA 12,0.

\section{Aspectos éticos}

Se ha considerado la Declaración de Helsinki de la Asociación Médica Mundial ${ }^{(23) .}$

\section{RESULTADOS}

Respecto al número de casos nuevos de COVID-19, las provincias de Huánuco, Leoncio Prado y Puerto Inca fueron las que presentaron el mayor número de nuevos casos, mientras que las provincias que presentaron el menor número de nuevos casos fueron Lauricocha, Yarowilca y Huacaybamba En lo que se refiere a la densidad poblacional, se utilizó la tasa de incidencia, encontrando que las provincias de Puerto Inca, Leoncio Prado y Huánuco fueron las que presentaban las más altas tasas de incidencia, mientras que las provincias de 


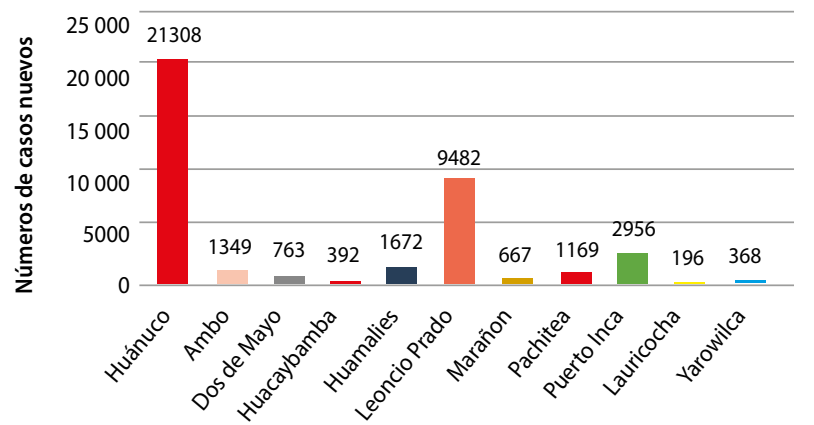

Provincias de Huánuco

Figura 1. Casos nuevos de COVID-19, Huánuco, 2020

Lauricocha, Yarowilca y Marañón presentaban las más bajas (ver Tabla 1).

Al evaluar la pobreza en el departamento de Huánuco, se tomó en cuenta la clasificación de los niveles de pobreza de cada distrito realizada en el Mapa de Pobreza Monetaria Provincial y Distrital 2018 del INEI y se agruparon por provincias, considerando la sumatoria de los niveles de pobreza de cada distrito. Se observa que la Provincia de Leoncio Prado tiene los distritos más pobres, seguida de la provincia de Huánuco y Lauricocha. Por otro lado, las provincias que tienen los

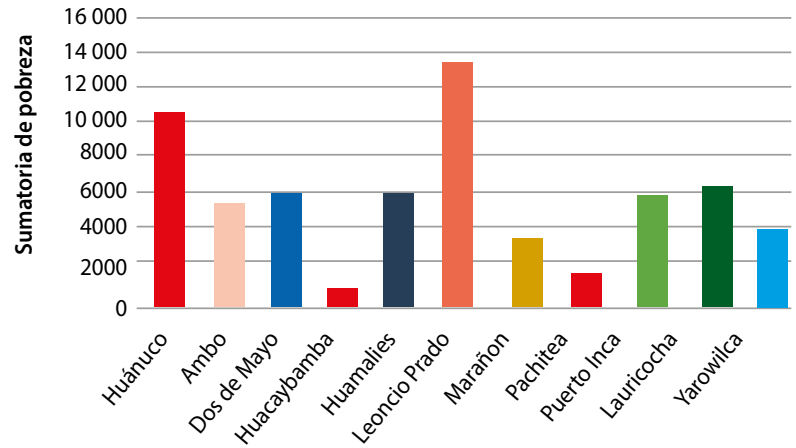

Provincias de Huánuco

Figura 2. Niveles de pobreza, Huánuco, 2018

distritos menos pobres son Huacaybamba, Pachitea y Marañón (ver Tabla 2).

Se determinó correlación positiva estadísticamente significativa de 0,646 para una $p<0,05$ entre el nivel de pobreza y la incidencia de la COVID-19 en Huánuco, durante el 2020 (ver Tabla 3).

Respecto a la gráfica de dispersión se aprecia una tendencia directa positiva ascendente, lo cual indica que a mayor nivel de pobreza mayor incidencia de COVID-19 (ver Figura 3).
Tabla 1. Incidencia de la COVID-19 en Huánuco, 2020

\begin{tabular}{lccc}
\hline Provincia & $\begin{array}{c}\text { Número de casos } \\
\text { nuevos }\end{array}$ & Población & $\begin{array}{c}\text { Tasa de } \\
\text { incidencia } \\
\text { (x 100 mil hab) }\end{array}$ \\
\hline Huánuco & 21308 & 315799 & 6,74 \\
Ambo & 1349 & 53247 & 2,53 \\
Dos de mayo & 763 & 32427 & 2,35 \\
Huacaybamba & 392 & 16372 & 2,39 \\
Huamalíes & 1672 & 52095 & 3,20 \\
Leoncio Prado & 9482 & 138275 & 6,85 \\
Marañón & 667 & 29160 & 2,28 \\
Pachitea & 1169 & 50086 & 2,33 \\
Puerto Inca & 2956 & 36987 & 7,99 \\
Lauricocha & 196 & 17114 & 1,14 \\
Yarowilca & 368 & 18705 & 1,96 \\
\hline
\end{tabular}

Tabla 2. Nivel de pobreza según sumatoria regional

\begin{tabular}{lc}
\hline Provincia & Sumatoria de pobreza \\
\hline Huánuco & 11164 \\
Ambo & 6054 \\
Dos de mayo & 6615 \\
Huacaybamba & 1211 \\
Huamalíes & 6487 \\
Leoncio Prado & 14045 \\
Marañón & 4000 \\
Pachitea & 2035 \\
Puerto Inca & 6499 \\
Lauricocha & 7054 \\
Yarowilca & 4586 \\
\hline
\end{tabular}


Tabla 3. Correlación entre el nivel de pobreza y la Incidencia de COVID-19 en Huánuco, 2020

\begin{tabular}{lc}
\hline Correlación & p - valor \\
\hline Rho de Spearman $\leq 0,05$ & 0,646 \\
\hline
\end{tabular}

\section{DISCUSIÓN}

Nuestros resultados demuestran que existe relación significativa entre la tasa de incidencia de la COVID-19 y la pobreza en Huánuco, durante el año 2020, en que se dio la primera ola de esta pandemia, evidenciando la realidad de unas de las regiones más pobres y menos competitivas del país ${ }^{(11)}$. Además, esta región presenta de la enorme dificultad en que se desenvuelve el sector sanitario local, principalmente en el primer nivel de atención, donde la promoción y la prevención de la salud se ven influenciados negativamente por los determinantes sociales, como el acceso al agua, a los servicios de desagüe y a infraestructura básica de vivienda, sumando las barreras educativas y culturales que padece la población pobre de ésta región e incrementando así los riesgos de contagio de la COVID-19.

En el Perú se dan las mismas condiciones en varios departamentos, por lo que el gobierno central decretó medidas que buscaron mitigar el contagio masivo y la afectación de poblaciones vulnerables a través de la imposición de una cuarentena temprana, cierre de fronteras, restricción de viajes nacionales e internacionales, distanciamiento social, otorgamiento de bono de apoyo social a poblaciones en pobreza y extrema pobreza, retiro adelantado de fondos de sistemas de pensiones privados y cierre de escuelas y universidades, en un afán por evitar el colapso de unos servicios de salud; de por sí debilitados ${ }^{(17,18)}$.

A nivel nacional, el Instituto Peruano de Economía menciona que en el 2019 existían aproximadamente 958000 personas a los que se les haría muy difícil conseguir una canastilla minúscula de víveres. Asimismo, aproximadamente 200000 peruanos ingresaron a la proporción real de miseria. La economía nacional obtuvo un débil efecto de desarrollo anualizado, siendo la más baja de estos últimos 10 años con un 2,2\%, haciendo más dificultoso luchar contra los inconvenientes sociales, tales como son la escasez y la escasez extrema ${ }^{(10)}$. A nivel regional, las regiones de Huancavelica, Cajamarca y Huánuco son las más pobres y las menos competitivas del país, según el último Índice de Competitividad Regional - INCORE 2019, elaborado por el Instituto Peruano de Economía (11). Para el 2019, el INCORE midió 40 indicadores agrupados en seis pilares fundamentales

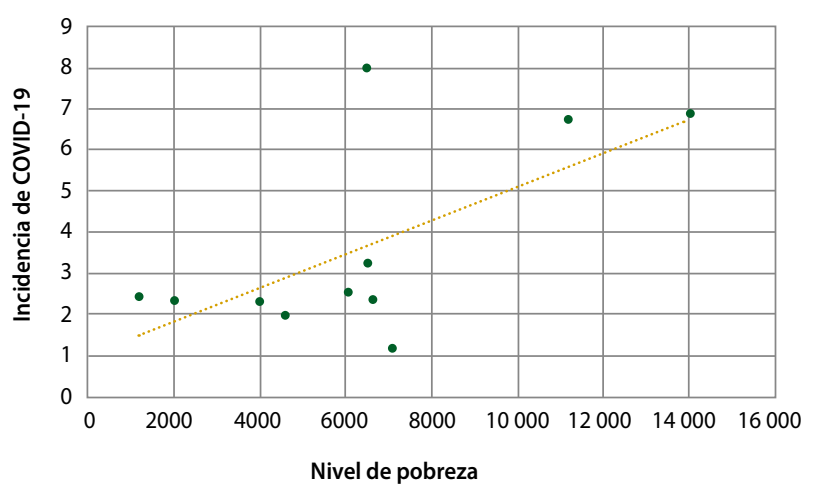

Figura 3. Gráfico de correlación entre la incidencia de la COVID-19 y el nivel de pobreza en Huánuco, 2020

(entorno económico, infraestructura, salud, educación, laboral e instituciones), los cuales se aplicaron a las 25 regiones del país ${ }^{(11)}$.

En cuanto a la pobreza, América Latina se encuentra como la región más desigual del planeta. Según informe de la Comisión Económica para América Latina y el Caribe (CEPAL), en el año 2018 "el 30,2 \% de los latinoamericanos son pobres y el $10,2 \%$, pobres extremos" $(15,16)$; lo que significa que gran parte de la población latinoamericana no cuenta con los recursos elementales para una vida digna ${ }^{(15)}$.

En Perú, según el Instituto Nacional de Estadística e Informática (INEI) por los resultados de la Encuesta Nacional de Hogares (ENAHO) del año 2019, el índice de pobreza monetaria afectó al $20,2 \%$ de la población del país, por lo que mantiene los mismos niveles del año 2018. Se considera población en condición de pobreza aquella cuyo gasto per cápita es inferior al valor de la línea de pobreza, que es el equivalente monetario de una canasta básica de consumo alimentario y no alimentario ${ }^{(19)}$.

Por su parte, Huánuco tenía, al 2019 el 3,6 \% de pobreza monetaria total, el $3,6 \%$ de incidencia de pobreza monetaria extrema y el $46,6 \%$ de la población en vulnerabilidad hacia la pobreza. Todos estos indicadores cayeron al año 2020, como producto de la pandemia de COVID-19, presentando un panorama sombrío de la economía a mediano plazo ${ }^{(20)}$.

En 2020, la economía peruana registró la peor caída desde 1989, al contraerse en un $11,1 \%$ debido al impacto de la pandemia. Por su parte, la región Huánuco experimentó una caída ligeramente menor (-10,9\%), explicada principalmente por la reducción de la actividad minera y de transportes ${ }^{(21)}$. 
Las restricciones a la libertad personal y económica que se requieren para enfrentar la emergencia sanitaria han significado, para muchos hogares, un corte abrupto de sus posibilidades de generar ingresos. En el corto plazo, esta situación tendrá impactos que, dependiendo de la duración de la emergencia, podrían traducirse en impactos mayores en el mediano plazo. Para los hogares también la situación es de una gran incertidumbre ${ }^{(22)}$.

La situación descrita no hace sino exacerbar las condiciones y factores de pobreza en la Región Huánuco, lo cual incidiría negativamente en el contagio de la población, incrementando las infecciones y reinfecciones con la Covid-19, condición que elevaría la mortalidad mientras no se logré la ansiada vacunación a los ciudadanos de este departamento y del Perú.

La presente investigación se desarrolló en el período de la primera ola de la pandemia de Covid-19 en la Región Huánuco, de marzo a diciembre de 2020, sin embargo, el escenario epidemiológico para las siguientes olas pandémicas se estima más grave. En ese sentido, consideramos prioritario el fortalecimiento de las acciones del Estado en su rol de cuidado y protección de la salud y su trabajo primordial sobre los determinantes sociales de la salud y las demás condiciones que genera la pobreza en la Región Huánuco.

\section{REFERENCIAS}

1. Córdova-Aguilar A, Rossani G. COVID-19 revisión de la literatura y su impacto en la realidad sanitaria peruana. Rev. Fac. Med. Hum. [Internet]. 2020 Jul [Consultado $2021 \mathrm{Abr} 11]$; 20(3): 471-477. Disponible en: http:// www.scielo.org.pe/scielo.php?script=sci_arttext\&pid=S2308-05312020000300471\&lng=es. http://dx.doi. org/10.25176/rfmh.v20i3.2984

2. Organización Mundial de la Salud (OMS). Neumonía de causa desconocida - China [Internet]. Ginebra: OMS; 2020 [Consultado 2021 Abr 11]. Disponible en: http:// www.who.int/csr/don/05-january-2020-pneumonia-ofunkown-cause-china/es/

3. Gil R, Bitar P, Deza C, Dreyse J, Florenzano M, Ibarra C, Jorquera J, Melo J, Olivi H, Parada MT, Rodríguez JC, Undurraga A, cuadro clínico del covid-19. Revista Médica Clínica Las Condes [Internet]. 2021 [Consultado 2021 Abr 11]; 32(1): 20-29. Disponible en: https://doi. org/10.1016/j.rmclc.2020.11.004

4. Organización Mundial de la Salud (OMS). Director-General's remarks at the media briefing on 2019-nCoV on 11 February 2020 [Internet]. Ginebra: OMS; 2020 [Consultado 2021 Abr 11]; Disponible en: https://www.who.int/ $\mathrm{dg} /$ speeches/detail/who-director-general-s-remarks-atthe-media-briefing-on-2019-ncov-on-11-february-2020

5. Paules $\mathrm{Cl}$, Marston HD, Fauci AS. Coronavirus Infections-More Than Just the Common Cold. JAMA [Internet]. 2020 [Consultado 2021 Abr 11]; Disponible en: https:// jamanetwork.com/journals/jama/fullarticle/2759815
6. Ubillús M. Coronavirus: vencer la epidemia dependerá de la vocación sanitaria y la responsabilidad social de la ciudadanía. Revista Peruana de Ciencias de la Salud [Internet]. 2020 Mar 3 [Consultado 2021 Abr 11]; 2(1): e84. https://doi.org/10.37711/rpcs.2020.2.1.84

7. Redacción EC. Coronavirus en Perú: ministra de salud confirma dos nuevos casos en el país, ambos en Huánuco. Diario El Comercio 2020 Mar 10 [Internet] [Consultado 2021 Abr 7]. Disponible en: https://elcomercio. pe/peru/coronavirus-en-peru-ministra-de-salud-confirma-dos-nuevo-casos-en-el-pais-ambos-en-huanuco-nndc-noticia/?ref=ecr

8. Ruiz-Aquino M, Díaz-Lazo AV, Ubillús M, Aguí-Ortiz AK, Rojas-Bravo V. Percepción de conocimientos y actitudes frente a COVID-19 en ciudadanos de la zona urbana de Huánuco. Rev. Fac. Med. Hum. [Internet]. 2021 Abr [Consultado 2021 Abr 11]; 21(2): 292-300. http://dx.doi. org/10.25176/rfmh.v21i1.3352

9. Noticia ONU. La pandemia de COVID-19 representa una "doble crisis" para los más pobres [Internet]. Organización de las Naciones Unidas; 2020 Oct 17 [Consultado 2021 Abr 7]. Disponible en: https://news.un.org/es/ story/2020/10/1482552

10. Merino M, Córdova JW, Aguirre JM, García AJ, López KE. Nivel de percepción sobre la pobreza en el Perú, causas y efectos sociales. Universidad y Sociedad [Internet]. 2020

11. La República. Huánuco, Cajamarca y Huancavelica: Las Regiones más pobres y menos competitivas [Internet]. Lima: Instituto Peruano de Economía; 2019 Jun 5 [Consultado 2021 Abr 7]. Disponible en:

12. Reporte De Casos Confirmados - Huánuco Covid19 [Internet]. Huánuco: Dirección Regional de Salud Huánuco; 2021 Ene 4 [Consultado $2021 \mathrm{Abr} 7$ ]. Disponible en: https://www.diresahuanuco.gob.pe/portal/covid19.htm

13. Instituto Nacional de Estadística e Informática (INEI). Mapa de Pobreza Monetaria Provincial y Distrital 2018 [Internet] [Consultado $2021 \mathrm{Abr}$ 7]. Disponible en: https://www.inei.gob.pe/media/MenuRecursivo/publicaciones_digitales/Est/Lib1646/libro.pdf

14. Pagano M, Gauvreau K. Fundamentos de Bioestadística. 2a ed. México DF: Kimberlee Thomson Learning; 2001

15. Molina N, Mejias ML. Impacto social de la COVID-19 en Brasil y Ecuador: donde la realidad supera las estadísticas. EDUMECENTRO [Internet]. 2020 Sep [Consultado 2021 Abr 12]; 12(3): 277-283. Disponible en: http:// scielo.sld.cu/scielo.php?script=sci_arttext\&pid=S207728742020000300277\&Ing=es. Epub 22-Jun-2020

16. Comisión Económica para América Latina y el Caribe (CEPAL). Panorama social de América Latina [Internet] [Consultado 2020 May 25]. Disponible en: https://repositorio.cepal.org/bitstream/handle/11362/44395/11/ S1900051_es.pdf

17. Aguilar-León P, Cotrina-Castañeda J, Zavala-Flores E. Infección por SARS-CoV-2 y tuberculosis pulmonar: análisis de la situación en el Perú. Cad. Saúde Pública [Internet]. 2020 [Consultado 2021 Abr 12]; 36(11): e00094520. Disponible en: http://www.scielo.br/scielo.php?script=sci_arttext\&pid=S0102-311X2020001100502\&lng=en. Epub Dec 18, 2020. https://doi.org/10.1590/0102-311x00094520

18. Amazonas Explorer. Coronavirus in Peru: the latest updates [Internet]. Cusco: Amazonas Explorer; 2021[Consultado 2021 Abr 7]. Disponible en: https:// 
amazonas-explorer.com/is-there-coronavirus-in-peru/\#Timeline_of_coronavirus_cases_in_Peru

19. Instituto Nacional de Estadística e Informática (INEI). Pobreza monetaria alcanzó al 20,2\% de la población en el año 2019 [Internet]. Lima: INEl; 2020 May 20 [Consultado 2021 Abr 7]. Disponible en: https://www.inei.gob.pe/ prensa/noticias/pobreza-monetaria-alcanzo-al-202-dela-poblacion-en-el-ano-2019-12196/

20. Ministerio de Desarrollo e Inclusión Social. Reporte regional de indicadores sociales del Departamento de Huánuco. Elaborado por la Dirección General de Seguimiento y Evaluación-DGSE-MIDIS [Internet] [Consultado 2021 Abr 12]. Disponible en: https://sdv.midis.gob.pe/ Redlnforma/Upload/regional/Huanuco.pdf
21. Economía de Huánuco cayó $10.9 \%$ el 2020 [Internet]. Lima: Instituto Peruano de Economía; 2020 [Consultado 2021 Abr 12]. Disponible en: https://www.ipe.org. pe/portal/economia-de-huanuco-cayo-10-9-en-2020/

22. Jaramillo $M$, Ñopo H. El impacto del Covid-19 sobre la economía peruana. Economíaunam [Internet]. 2020 [Consultado 2021 Abr 13]; 17(51): 136-46. Disponible en: https://www.grade.org.pe/publicaciones/el-impacto-del-covid-19-sobre-la-economia-peruana/

23. Principios éticos para las investigaciones médicas en seres humanos (Declaración de Helsinki de la Asociación Médica Mundial) [Internet]. Fisterra; 2003 [Consultado 2021 Abr 11]. Disponible en: https://www.fisterra.com/ formacion/bioetica/principios-eticos-para-investigaciones-medicas-seres-humanos-declaracion-helsinki-asociacion-medica-mundial/ 